\title{
The 7th National Conference on Bioinformatics and Systems Biology of China
}

\author{
Zhirong Sun $^{1, *}$, Kui Hua ${ }^{2}$, Xuegong Zhang ${ }^{1,2}$, Feng-Biao Guo ${ }^{3}$ and Jian Huang ${ }^{4}$ \\ 1 MOE Key Laboratory of Bioinformatics, School of Life Sciences, Tsinghua University, Beijing 100084, China \\ 2 MOE Key Laboratory of Bioinformatics; Bioinformatics Division and Center for Synthetic \& Systems Biology, TNLIST; \\ Department of Automation, Tsinghua University, Beijing 100084, China \\ ${ }^{3}$ Bioinformatics Center in School of Life Science and Technology, University of Electronic Science and Technology of China, \\ Chengdu 610054, China \\ ${ }^{4}$ Center of Bioinformatics (COBI), Key Laboratory for Neuro Information of Ministry of Education, University of Electronic \\ Science and Technology of China, Chengdu 610054, China \\ * Correspondence: sunzhr@mail.tsinghua.edu.cn
}

Received November 4, 2016

The 7th National Conference on Bioinformatics and System Biology and the International Workshop on Advanced Bioinformatics and Precision Medicine was successfully held in Chengdu, Sichuan Province, China, on October 7-9, 2016. The conference is organized by Chinese Society for Cell Biology (CSCB), National Natural Science Foundation of China (NSFC), and MOE Key Laboratory of Bioinformatics, Tsinghua University, and hosted by University of Electronic Science and Technology of China (UESTC). Prof. Runsheng Chen of Institute of Biophysics, CAS, is the organizing chair of the conference, and Prof. Zhirong Sun of Tsinghua University is the general secretary. Professors Feng-Biao Guo and Jian Huang of UESTC led the local organization team. A total of about 1,047 professionals and graduate students from all over the country and several US universities attended the conference.

The theme of this year's conference is "Bioinformatics and Big Data and Precision Medicine". The conference features 16 invited plenary talks, 30 invited session talks, 43 contributed talks and 94 poster presentations. A total of 255 abstracts are included in the conference proceedings, covering the topic of structure and function of protein and other biological macromolecules, identification and functional analysis of non-coding RNAs, transcriptional regulation and epigenetics, new concepts, new ideas and trends of development of bioinformatics, sequence information mining and molecular recognition, development and application of sequencing technology in the era of big data and so on.

\section{The INVITED PLENARY TALKS}

The conference features 16 invited plenary talks from world leaders in biology, life science and bioinformatics. All invited plenary talks are arranged in the mornings. In the first morning, Academician of CAS, Prof. Bolin Hao of Fudan University, gave the first talk on microbial classification problems. Academician of CAS, Prof. Runsheng Chen of the Institute of Biophysics talked about the concept of Precision Medicine. He pointed out the opportunities and challenges in Precision Medicine by giving the example of non-coding RNA analysis. Prof. Bin Ren of University of California, San Diego, talked about their work on long-range chromatin interactions, which contribute novel insights into a broad spectrum of human traits and diseases. Prof. Ying Xu of University of Georgia shared an interesting and insightful view on ATP syntheses in cancer based on the chemical reaction called Fenton reaction. Prof. Hao Li of University California, San Francisco, presented their statistical frameworks and computational tools to uncover the genotype-phenotype relation, which integrated with genome-wide association study (GWAS) data may help to discover common genetic mechanisms underlying seemingly un-related human traits. The second day of the conference began with the 


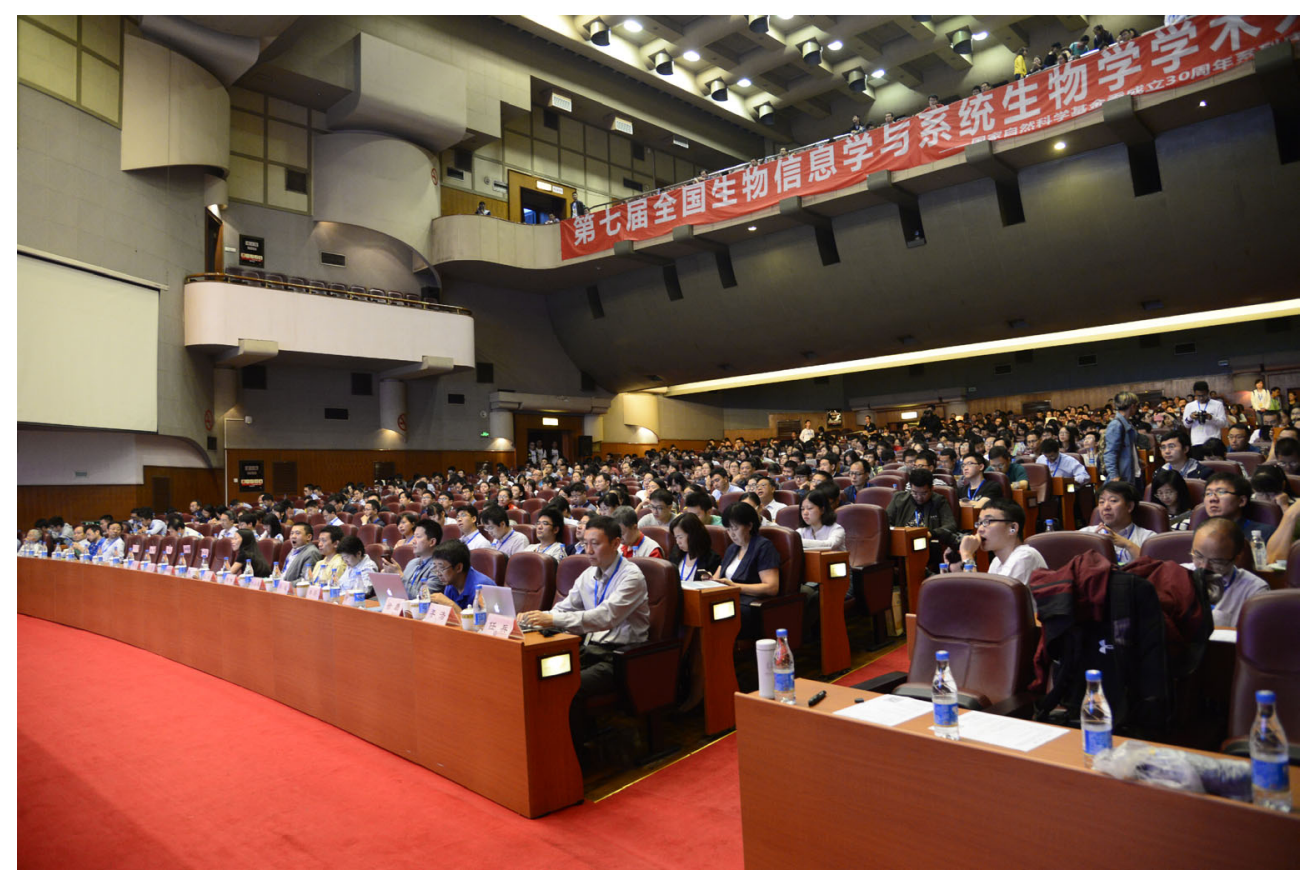

\section{A snapshot of the meeting}

invited plenary talk by Prof. Chao Tang of Peking University, with the title "Growth behavior of microbes on mixed carbon sources: Monod's problem revisted". Prof. Wei Li of Baylor College of Medicine shared his novel biological insights in cancer epigenome by using existing big data. Dr. Jiashun Zheng of University California, San Francisco, talked about cellular aging in the view of system biology. Prof. Jingdong Han of PICB, Shanghai Institute of Biological Sciences, CAS, introduced a systems approach to reverse engineer lifespan extension by dietary restriction. Prof. Sheng Zhong of University of California, San Diego, presented a search engine for web-based functional genomic data. Prof. Wei

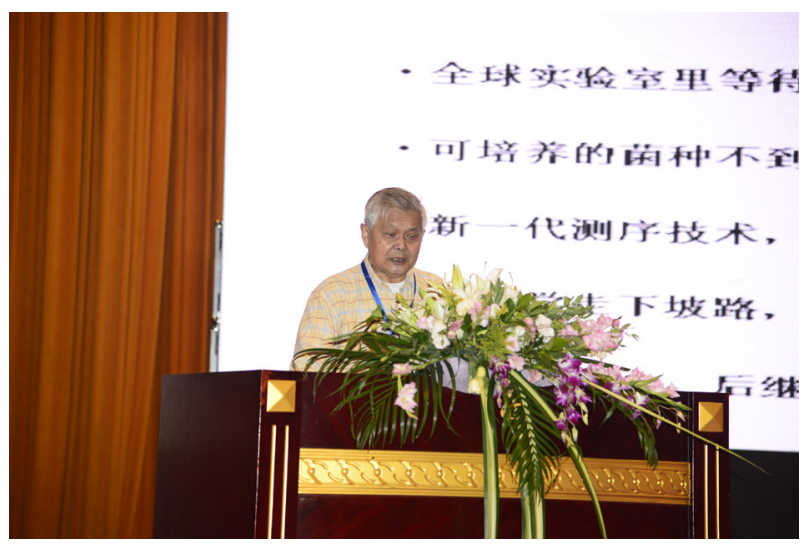

Keynote talk by Academician of CAS, Prof. Bolin Hao
Xie of Tsinghua University shared their latest discovery on epigenetic inheritance and reprogramming of chromatin modifications in early mammalian development. In the last morning of the conference, Prof. Michael Q. Zhang of University of Texas, Dallas and Tsinghua University first showed their new technologies for studying 3D cancer genomes. Prof. Xiaole Shirley Liu of Harvard University and Tongji University then presented a new method to infer tumor immunity and T-cell receptor repertoire from TCGA RNA-seq data. Prof. Zhiping Weng of University of Massachusetts Medical School reported their results on annotating non-coding variants by expanding the ENCODE encyclopedia. Prof. Kun Huang of Ohio State University talked about integrative analysis of imaging and genomic data. Prof. Ting Wang of Washington University in St. Louis gave the last talk of the conference. He presented their results on epimutations in cancer cells and showed that cancer cells undergo identity crisis. This finding has potential implications in understanding the epigenetic's impact on Precision Medicine.

\section{INVITED SESSION TALKS, CONTRIBUTED TALKS AND POSTER PRESENTATIONS}

Besides invited plenary talks in the mornings, there are parallel sessions in the afternoons with invited session talks and contributed talks. Invited session talks are arranged in the first half of the afternoons and contributed 


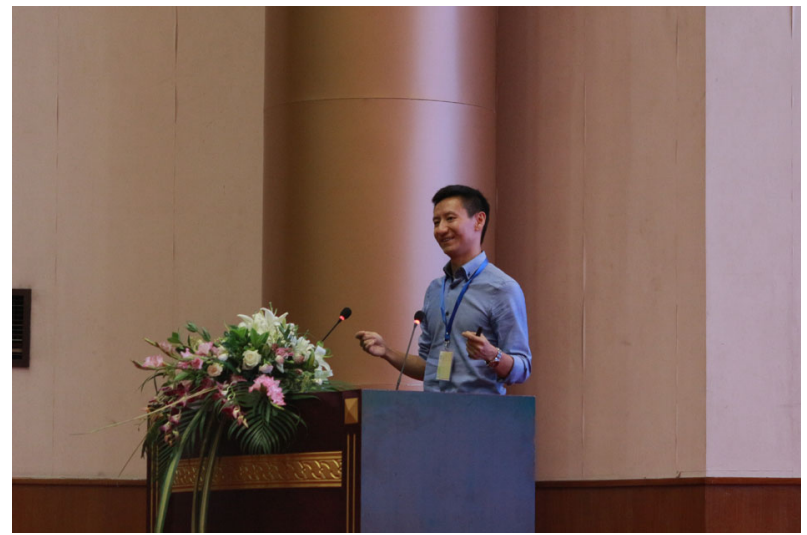

Keynote talk by Prof. Sheng Zhong

talks in the second half. A total of 73 talks were given in the invited and contributed sessions around 7 major topics: (i) structure and function of protein and other biological macromolecules, (ii) systems biology of complex biological process and complex diseases, (iii) identification and functional analysis of non-coding RNAs, (iv) transcriptional regulation and epigenomic modification, (v) new concepts, ideas and trends in bioinformatics, (vi) modeling and functional analysis of biological networks and systems, (vii) sequencing applications in the big data era.

Poster presentation was on the evening of the first day. Nearly 100 posters were displayed. The freedom of discussion and the flexibility of time made it a good opportunity for interaction and exchange of ideas from different researchers.

\section{AWARDS}

With the generous donation of some sponsors, the 2016 National Conference on Bioinformatics and System Biology was able to set three tracks of awards to conference presenters. Zhi Lu from Tsinghua University won the first prize of oral lecture with his excellent oral lecture of "Predict noncoding RNA's secondary structures by integrating high-throughput probing data and energy model". The second prizes of oral lecture go to Zhiwei Cao from Tongji University and $\mathrm{Li} \mathrm{Li}$ from Huazhong Agricultural University. Their lectures' titles are "The computational methods progresses for antibody and antigen specifically recognization" and "Comparative chromatin organization revealed by HiC experiment" respectively. The third prizes go to Kiu Fai Au from the University of Iowa, Li Yang from Shanghai Institutes for Biological Sciences and Kang Ning from Huazhong University of Science and Technology. Their lectures' titles are "Determination of phased genotypes and allelespecific expression at isoform level by hybrid sequencing", "Complex circRNA biogenesis by alternative back-splicing" and "Big-data for microbiome researches: method development and the applications in healthcare and environment", respectively. Three tracks of awards to excellent poster: the doctor candidate named Hong-Yi Zhang from Harbin Medical University won the first prize by his excellent poster titled "Integrating multi-dimensional genomic data to identify core gene modules and cooperative networks in human cancers". The second prizes of excellent poster go to Kang Zhang from China Agricultural University and Chenfei Wang from Tongji University, whose posters are "Pathogenicity in Ustilaginoidea virens revealed by a predicted protein-protein networks" and "Distinct features of H3K4me3 and $\mathrm{H} 3 \mathrm{H} 27$ me3 chromatin domains in pre-implantation embryos" respectively. Bingqiang Liu from Shandong University and Xin Sheng from Beijing Institute of genome research, Chinese Academy of Sciences and Le $\mathrm{Xu}$ from Tsinghua University won the third prizes. Their posters are "Development of computational tools in DNA motif identification and analyses", "MTD: a mammalian transcriptomic database to explore gene expression and regulation" and "Epigenetic regulation of gene transcription by RNA-directed DNA methylation in rice", respectively. 